\title{
Witten's Gauge Field Equations and an Infinite-Dimensional Grassmann Manifold
}

\section{Norio Suzuki}

Research Institute for Mathematical Sciences, Kyoto University, Kyoto, 606 Japan

\begin{abstract}
Witten's gauge fields are interpreted as motions on an infinitedimensional Grassmann manifold. Unlike the case of self-dual Yang-Mills equations in Takasaki's work, the initial data must satisfy a system of differential equations since Witten's equations comprise a pair of spectral parameters. Solutions corresponding to (anti-) self-dual Yang-Mills fields are characterized in the space of initial data and in application, some Yang-Mills fields which are not self-dual, anti-self-dual nor abelian can be constructed.
\end{abstract}

\section{Introduction}

Consider a gauge field $\nabla$ in the eight-dimensional complex space $\mathbb{C}^{8}$ satisfying

$$
\begin{aligned}
& {\left[\nabla_{y_{\mu}}, \nabla_{y_{v}}\right]=(1 / 2) \sum_{\alpha=0}^{3} \sum_{\beta=0}^{3} \varepsilon_{\mu v \alpha \beta}\left[\nabla_{y_{\alpha}}, \nabla_{y_{\beta}}\right],} \\
& {\left[\nabla_{z_{\mu}}, \nabla_{z_{v}}\right]=(-1 / 2) \sum_{\alpha=0}^{3} \sum_{\beta=0}^{3} \varepsilon_{\mu v \alpha \beta}\left[\nabla_{z_{\alpha}}, \nabla_{z_{\beta}}\right],} \\
& {\left[\nabla_{y_{\mu}}, \nabla_{z_{v}}\right]=0, \quad(\mu, v=0,1,2,3),}
\end{aligned}
$$

where $(y, z)=\left(y_{0}, y_{1}, y_{2}, y_{3}, z_{0}, z_{1}, z_{2}, z_{3}\right)$ are coordinates of $\mathbb{C}^{8}, \nabla_{y_{\mu}}$ and $\nabla_{z_{\mu}}$ are covariant derivatives, and $\varepsilon_{\mu v \alpha \beta}$ denotes the totally antisymmetric tensor such that $\varepsilon_{0123}=1$.

Set $x=(y+z) / 2, w=(y-z) / 2$. Witten [9] pointed out that Eq. (0.1) imply the full Yang-Mills equations

$$
\sum_{\mu=0}^{3}\left[\nabla_{x_{\mu}},\left[\nabla_{x_{\mu}}, \nabla_{x_{\nu}}\right]\right]=0 \quad(v=0,1,2,3)
$$

on the diagonal subspace $\Delta=\left\{(y, z) \in \mathbb{C}^{8} \mid w=0\right\}$, and further, that a gauge field on $\Delta$ satisfies ( 0.2$)$ if and only if it can be extended to a neighborhood of $\Delta$ consistently to $(0.1) \bmod \left(w_{0}, w_{1}, w_{2}, w_{3}\right)^{2}$. Here $\left(w_{0}, w_{1}, w_{2}, w_{3}\right)^{2}$ denotes the square of the ideal generated by $w_{0}, w_{1}, w_{2}$, and $w_{3}$. 
In this paper, we rewrite $(0.1)$ in the language of Sato's soliton theory $[4,5]$ and investigate the structure of the solution space of (0.1) on the analogy of Takasaki's work on self-dual Yang-Mills fields [7,8]: we solve an initial-value problem of differential equations with respect to functions with values in an infinite-dimensional Grassmann manifold (see Theorem 2).

In our case, there appear a pair of spectral parameters $\lambda_{1}, \lambda_{2}$. The main difference from the case of one spectral parameter is that the initial data must satisfy a system of differential equations if the problem is solvable (see Proposition 5 and cf. Takasaki $[7,8])$.

Through the restriction to the diagonal space $\Delta$, the totality of gauge fields satisfying (0.1) can be regarded as a class of Yang-Mills fields including all the selfdual or anti-self-dual fields. From our point of view, it is interesting to characterize self-dual or anti-self-dual fields in terms of initial data. In fact, a simple characterization is obtained (see Sect. 3) and in application, we shall construct an example of Yang-Mills fields which are neither self-dual nor anti-self-dual (see Sect. 4).

The announcement of our results [6] was already published in 1984. Ueno treated the same problem independently and gave it a cohomological formulation (unpublished).

Notations. We shall use the following standard notations: $\mathbb{N}$ denotes the set of nonnegative integers. $\mathbb{Z}$ denotes the set of integers. $\mathbb{C}$ denotes the complex number field. $M_{n}(\mathbb{C})$ denotes the total matrix algebra. 1 denotes the unit matrix of size $n \times n$. Let $R$ be a ring. Then we denote by $R[x]$ the ring of polynomials of $x$ with coefficients in $R$, and denote by $R[[x]]$ the ring of formal power series of $x$ with coefficients in $R$.

\section{Linearization}

Set $x_{11}=y_{0}+\sqrt{-1} y_{1}, t_{11}=y_{2}+\sqrt{-1} y_{3}, x_{12}=y_{2}-\sqrt{-1} y_{3}, t_{12}=-y_{0}+\sqrt{-1} y_{1}$, $x_{21}=z_{0}+\sqrt{-1} z_{1}, t_{21}=z_{2}-\sqrt{-1} z_{3}, x_{22}=z_{2}+\sqrt{-1} z_{3}$, and $t_{22}=-z_{0}+\sqrt{-1} z_{1}$. Then, introducing parameters $\lambda_{1}, \lambda_{2}$, we can rewrite $(0.1)$ as follows:

$$
\left[-\lambda_{a} \nabla_{x_{a b}}+\nabla_{t_{a b}},-\lambda_{c} \nabla_{x_{c d}}+\nabla_{t_{c d}}\right]=0 \quad(a, b, c, d=1,2) .
$$

Throughout this paper we discuss in the category of formal power series. Hence the gauge potentials $A_{t_{a b}}, A_{x_{a b}}$ belong to the ring of formal power series with matrix coefficients $M_{n}(\mathbb{C})[[t, x]]$, where $\nabla_{t_{a b}}=\partial_{t_{a b}}+A_{t_{a b}}, \nabla_{x_{a b}}=\partial_{x_{a b}}+A_{x_{a b}}$, $t=\left(t_{11}, t_{12}, t_{21}, t_{22}\right)$, and $x=\left(x_{11}, x_{12}, x_{21}, x_{22}\right)$.

Now we "fix" the gauge, namely, restrict the freedom of gauge so that $A_{x_{a b}}=0$ for $a, b=1,2$. (The gauge-fixing is analogous to that of Chau et al. [1] and Pohlmeyer [3] for self-dual Yang-Mills equations.) Then (1.1) reads

$$
\left[-\lambda_{a} \partial_{x_{a b}}+\nabla_{t_{a b}},-\lambda_{c} \partial_{x_{c d}}+\nabla_{t_{c d}}\right]=0 \quad(a, b, c, d=1,2) .
$$

More precisely, we have

Proposition 1. For any $\nabla$ satisfying (0.1), there exists a gauge transformation $\nabla \rightarrow \tilde{\nabla}=g^{-1} \nabla g, g \in M_{n}(\mathbb{C})[[t, x]]$, such that $\tilde{\nabla}_{x_{a b}}=g^{-1} \nabla_{x_{a b}} g=\partial_{x_{a b}}$ for $a, b=1,2$. 
Proof. Equations (1.1) imply that $\left[\nabla_{x_{a b}}, \nabla_{x_{c d}}\right]=0$ for $a, b, c, d=1,2$, which are the integrability conditions for the linear equations

$$
\left(\frac{\partial}{\partial x_{a b}}+A_{x_{a b}}\right) g=0 \quad(a, b=1,2) \text {. }
$$

Thus for any $A_{x_{a b}} \in M_{n}(\mathbb{C})[[t, x]](a, b=1,2)$ satisfying (1.1) there exists a solution $g=\sum_{i, j, k, l \geqq 0} g_{i j k l} x_{11}^{i} x_{12}^{j} x_{21}^{k} x_{22}^{l}$ of (1.3) such that $g_{i j k l} \in M_{n}(\mathbb{C})[[t, x]]$ and $g_{0000}=\mathbb{1}$. This $g$ is invertible in $M_{n}(\mathbb{C})[[t, x]]$ and satisfies $g^{-1} \nabla_{x_{a b}} g=g^{-1}\left(\partial_{x_{a b}}+A_{x_{a b}}\right) g$ $=g^{-1} g \partial_{x_{a b}}+g^{-1}\left(\frac{\partial g}{\partial x_{a b}}+A_{x_{a b}} g\right)=\partial_{x_{a b}}$. q.e.d.

We shall investigate the structure of solutions to Eq. (1.2). First we note that the system of Eq. (1.2) is nothing but the integrability condition for the linear equations,

$$
\left(-\lambda_{a} \partial_{x_{a b}}+\partial_{t_{a b}}+A_{t_{a b}}\right) w(\lambda)=0 \quad(a, b=1,2) .
$$

Proposition 2. $A_{t_{a b}} \in M_{n}(\mathbb{C})[[t, x]](a, b=1,2)$ are solutions of $(1.2)$ if and only if there exists a solution $w(\lambda)=\sum_{i, j \geqq 0} w_{i j} \lambda_{1}^{-i} \lambda_{2}^{-j}$ of (1.4) such that $w_{00}=1$, namely, $w_{i j} \in M_{n}(\mathbb{C})\{[t, x]]$ which satisfy $w_{00}=1, w_{i j}=0$ if $i<0$ or $j<0$, and

$$
\begin{aligned}
& -\partial_{x_{1 b}} w_{i+1, j}+\left(\partial_{t_{1 b}}+A_{t_{1 b}}\right) w_{i j}=0, \\
& -\partial_{x_{2 b}} w_{i, j+1}+\left(\partial_{t_{2 b}}+A_{t_{2 b}}\right) w_{i j}=0,
\end{aligned}
$$

for any $i, j \in \mathbb{Z}, b=1,2$. Proof of sufficiency. Suppose that there exists $w(\lambda)=\sum_{i, j \geqq 0} w_{i j} \lambda_{1}^{-i} \lambda_{2}^{-j}$ satisfying
(1.4) such that $w_{00}=1$. Equations (1.4) imply that

$$
\left[-\lambda_{a} \partial_{x_{a b}}+\nabla_{t_{a b}},-\lambda_{c} \partial_{x_{c d}}+\nabla_{t_{c d}}\right] w(\lambda)=0 \quad(a, b, c, d=1,2)
$$

Note that the commutator is a differential operator of order 0 , namely, an element of $M_{n}(\mathbb{C})[[t, x]]$. Multiplying both sides of Eqs. (1.6) by $w(\lambda)^{-1}$ from the right, we obtain (1.2).

Proof of necessity. For any $i, j \in \mathbb{Z}$, consider a system of four equations

$$
\begin{aligned}
\left(E_{i j}\right) \quad-\partial_{x_{1 b}} w_{i j}+\left(\partial_{t_{1 b}}+A_{t_{1 b}}\right) w_{i-1, j}=0, \\
-\partial_{x_{2 b}} w_{i j}+\left(\partial_{t_{2 b}}+A_{t_{2 b}}\right) w_{i, j-1}=0 \quad(b=1,2),
\end{aligned}
$$

which is a part of the system of Eqs. (1.5). The integrability condition for the equations $\left(E_{i j}\right)$ with $w_{i j}$ as the unknown function is as follows:

$$
\begin{gathered}
\left(\partial_{x_{11}} \nabla_{t_{12}}-\partial_{x_{12}} \nabla_{t_{11}}\right) w_{i-1, j}=0 \\
\left(\partial_{x_{21}} \nabla_{t_{22}}-\partial_{x_{22}} \nabla_{t_{21}}\right) w_{i, j-1}=0 \\
\hat{\partial}_{x_{1 b}} \nabla_{t_{2 d}} w_{i, j-1}-\partial_{x_{2 d}} \nabla_{t_{1 b}} w_{i-1, j}=0 \quad(b, d=1,2) .
\end{gathered}
$$

Now we define $w_{00}=\mathbb{1}$ and $w_{i j}=0$ for any $i, j, \in \mathbb{Z}$ such that $i<0$ or $j<0$. Then $\left(E_{i j}\right)$ is trivially satisfied for $i=j=0$ and for any $i, j \in \mathbb{Z}$ such that $i<0$ or $j<0$. For 
$i, j \in \mathbb{N}$, we define $w_{i j}$ inductively. Assume that $\left\{w_{i j}\right\}_{i, j \in \mathbb{N}, l+\jmath \leqq m}$ are defined to satisfy $\left(E_{i j}\right)$ for any $i, j \in \mathbb{N}$ such that $i+j \leqq m$. (This assumption actually holds for $m=0$.) We shall prove that for any $i, j \in \mathbb{N}$ such that $i+j=m+1$, there exists $w_{i j}$ which satisfies $\left(E_{i j}\right)$. To prove this, it is sufficient to prove the integrability conditions $(1.7 \mathrm{a}),(1.7 \mathrm{~b})$ and $(1.7 \mathrm{c})$.

Proof of (1.7a). (i) Equations (1.2) imply $\left[\nabla_{t_{12}}, \partial_{x_{11}}\right]-\left[\nabla_{t_{11}}, \partial_{x_{12}}\right]=0$.

(ii) By the assumption, $w_{i-1, j}$ satisfies $\left(E_{i-1, j}\right)$. Especially,

$$
\hat{\partial}_{x_{1 b}} w_{i-1, j}=\nabla_{t_{1 b}} w_{i-2, j} \quad(b=1,2) .
$$

(iii) Equations (1.2) imply $\left[\nabla_{t_{12}}, \nabla_{t_{11}}\right]=0$.

It follows from (i), (ii), and (iii) that

$$
\begin{aligned}
\left(\partial_{x_{11}} \nabla_{t_{12}}-\partial_{x_{12}} \nabla_{t_{11}}\right) w_{i-1, j} & =\left(\nabla_{t_{12}} \partial_{x_{11}}-\nabla_{t_{11}} \partial_{x_{12}}\right) w_{i-1, j} \\
& =\left(\nabla_{t_{12}} \nabla_{t_{11}}-\nabla_{t_{11}} \nabla_{t_{12}}\right) w_{i-2, j} \\
& =\left[\nabla_{t_{12}}, \nabla_{t_{11}}\right] w_{i-2 . j} \\
& =0 .
\end{aligned}
$$

Equation (1.7b) can be derived in the same way.

Proof of $(1.7 \mathrm{c})$.

(i) Equations (1.2) imply $\left[\partial_{x_{1 b}}, \nabla_{t_{2 d}}\right]=\left[\partial_{x_{2 d}}, \nabla_{t_{1 b}}\right]=0$.

(ii) By the assumption, $w_{i, j-1}$ satisfies $\left(E_{i, j-1}\right)$ and $w_{i-1, j}$ satisfies $\left(E_{i-1, j}\right)$. Especially, we obtain

$$
\partial_{x_{1 b}} w_{i, j-1}=\nabla_{t_{1 b}} w_{i-1, j-1}, \quad \partial_{x_{2 d}} w_{i-1, j}=\nabla_{t_{2 d}} w_{i-1, j-1} .
$$

(iii) Equations (1.2) imply $\left[\nabla_{t_{2 d}}, \nabla_{t_{1 b}}\right]=0$.

It follows from (i), (ii), and (iii) that

$$
\begin{aligned}
\partial_{x_{1 b}} \nabla_{t_{2 d}} w_{i, j-1}-\partial_{x_{2 d}} \nabla_{t_{1 b}} w_{i-1, j} & =\nabla_{t_{2 d}} \partial_{x_{1 b}} w_{i, j-1}-\nabla_{t_{1 b}} \partial_{x_{2 d}} w_{i-1, j} \\
& =\nabla_{t_{2 d}} \nabla_{t_{1 b}} w_{l-1, j-1}-\nabla_{t_{1 b}} \nabla_{t_{2 d}} w_{i-1, j-1} \\
& =\left[\nabla_{t_{2 d}}, \nabla_{t_{1 b}}\right] w_{i-1, j-1} \\
& =0 .
\end{aligned}
$$

Thus we can obtain $\left\{w_{i j}\right\}_{l, j \in \mathbb{N}}$ satisfying (1.5) inductively (more precisely, by using Zorn's lemma). q.e.d.

When $i=j=0$, (1.5) reads

$$
-\partial_{x_{1 b}} w_{1,0}+A_{t_{1 b}}=0, \quad-\partial_{x_{2 b}} w_{0,1}+A_{t_{2 b}}=0 .
$$

Therefore, to solve Eqs. (1.2), it is sufficient to solve the equations

$$
\begin{aligned}
& -\partial_{x_{1 b}} w_{i+1, j}+\partial_{t_{1 b}} w_{i j}+\left(\partial_{x_{1 b}} w_{1,0}\right) w_{i j}=0 \\
& -\partial_{x_{2 b}} w_{i, j+1}+\partial_{t_{2 b}} w_{i j}+\left(\partial_{x_{2 b}} w_{0,1}\right) w_{i j}=0
\end{aligned}
$$

$(i, j \in \mathbb{Z}, b=1,2)$. 
More precisely, we have

Proposition 3. The relations (1.8) give a one-to-one correspondence between (i) solutions $A=\left(A_{t_{a b}}\right)_{a, b=1,2}$ to (1.2) and

(ii) equivalence classes of solutions $w(\lambda)=\sum_{i, j \geqq 0} w_{i j} \lambda_{1}^{-i} \lambda_{2}^{-j}$ to (1.9) such that $w_{00}=1$ modulo right-multiplication by $v(\lambda)=\sum_{i, j \geqq 0} v_{i j} \lambda_{1}^{-i} \lambda_{2}^{-j}$ satisfying $v_{00}=1$
and

$$
\left(-\lambda_{a} \partial_{x_{a b}}+\partial_{t_{a b}}\right) v(\hat{\imath})=0 \text { for } \quad a, b=1,2 .
$$

Proof. A surjection \{solutions $w(\lambda)$ of (1.9) such that $\left.w_{00}=1\right\} \rightarrow\{$ solutions $A$ of $(1.2)\}$ is established by Proposition 2. Now let $w(\lambda)$ and $\tilde{w}(\lambda)$ be solutions of (1.9) both corresponding to $A=\left(A_{t_{a b}}\right)_{a, b=1,2}$. Set $v(\hat{\lambda})=w(\lambda)^{-1} \tilde{w}(\lambda)$. Then we have

$$
\begin{aligned}
\left(-\lambda_{a} \partial_{x_{a b}}+\partial_{t_{a b}}\right) \vee(\lambda)= & \left(\left(-\lambda_{a} \partial_{x_{a b}}+\partial_{t_{a b}}\right) w(\lambda)^{-1}\right) \tilde{w}(\lambda) \\
& +w(\lambda)^{-1}\left(-\lambda_{a} \partial_{x_{a b}}+\partial_{t_{a b}}\right) \tilde{w}(\lambda) \\
= & w(\lambda)^{-1} A_{t_{a b}} \tilde{w}(\lambda)-w(\lambda)^{-1} A_{t_{a b}} \tilde{w}(\lambda) \\
= & 0 .
\end{aligned}
$$

If $w(\hat{\lambda}), \quad \tilde{w}(\hat{\lambda}) \in M_{n}(\mathbb{C})[[t, x]]\left[\left[\lambda_{1}^{-1}, \lambda_{2}^{-1}\right]\right]$ and $w_{00}=\tilde{w}_{00}=1$, then $v(\lambda)$ $=w(\lambda)^{-1} \tilde{w}(\lambda) \in M_{n}(\mathbb{C})[[t, x]]\left[\left[\lambda_{1}^{-1}, \lambda_{2}^{-1}\right]\right]$ and $v_{00}=\mathbb{1}$.

Conversely, let $v(\lambda) \in M_{n}(\mathbb{C})[[t, x]]\left[\left[\lambda_{1}^{-1}, \lambda_{2}^{-1}\right]\right]$ be a solution of $(1.10)$ such that $v_{00}=\mathbb{1}$, and let $w(\lambda) \in M_{n}(\mathbb{C})[[t, x]]\left[\left[\lambda_{1}^{-1}, \lambda_{2}^{-1}\right]\right]$ be a solution of (1.9) corresponding to $A$ such that $w_{00}=1$. Set $\tilde{w}(\lambda)=w(\lambda) v(\lambda)$. Then we obtain $w(\lambda) \in M_{n}(\mathbb{C})[[t, x]]\left[\left[\lambda_{1}^{-1}, \lambda_{2}^{-1}\right]\right], w_{00}=\mathbb{1}$, and

$$
\begin{aligned}
\left(-\lambda_{a} \partial_{x_{a b}}+\partial_{t_{a b}}\right) \tilde{\mathrm{w}}(\lambda) & =\left(-\lambda_{a} \partial_{x_{a b}}+\partial_{t_{a b}}\right)(\mathrm{w}(\lambda) \mathrm{v}(\lambda)) \\
& =\left\{\left(-\lambda_{a} \partial_{x_{a b}}+\partial_{t_{a b}}\right) \mathrm{w}(\lambda)\right\} \mathrm{v}(\lambda) \\
& =-A_{t_{a b}} w(\lambda) v(\lambda) \\
& =-A_{t_{a b}} \tilde{w}(\lambda) .
\end{aligned}
$$

Namely, $\tilde{w}(\dot{i})$ is a solution of (1.9) corresponding to $A$. q.e.d.

\section{Motions on an Infinite-Dimensional Grassmann Manifold}

Let $Z=\mathbb{Z} \times \mathbb{Z}, N=\mathbb{N} \times \mathbb{N}, N^{c}=Z \backslash N$ and $R$ be a ring with a unity $\mathbb{1}$. For any $w(\hat{\lambda})=\sum_{(\imath, j) \in Z} w_{i j} \lambda_{1}^{-i} \hat{\lambda}_{2}^{-J} \in R\left[\left[\lambda_{1}^{-1}, \lambda_{2}^{-1}\right]\right]$ such that $w_{00}=1$ and $w_{i j}=0$ for $(i, j) \in N^{c}$, define a matrix of infinite size $\xi=\left(\xi_{k l}^{i j}\right)_{(i, j), \in Z,(k, l) \in N^{c}}$ by the product of matrices $\left(w_{i-k, j-l}^{*}\right)_{(i, j) \in Z,(k, l) \in N^{c}}$ and $\left(w_{i-k, j-l}\right)_{(i, j) \in Z,(k, l) \in N^{c}}$, i.e. by $\xi_{k l}^{i j}$ $=\sum_{(g, h) \in N^{c}} w_{i-g, j-h}^{*} w_{g-k, h-l}$, where $w_{i j}^{*}$ are coefficients of $w^{-1}$, i.e. $w^{-1}$ $=\sum_{(i, j) \in Z} w_{i j}^{*} \lambda_{1}^{-i} \lambda_{2}^{-j}$. Then we obtain $\xi_{k l}^{i j}=\delta_{k}^{i} \delta_{l}^{j} \mathbb{1}$ if $(i, j) \in N^{c}, \xi_{k l}^{i j}=0$ if $i<k$ or 
$j<l$, and $\Lambda_{a} \xi=\xi C_{a}(a=1,2)$, where

$$
\begin{aligned}
& \Lambda_{1}=\left(\delta_{k}^{i+1} \delta_{l}^{j} \mathbb{1}\right)_{(i, j) \in Z,(k, l) \in Z}, \\
& \Lambda_{2}=\left(\delta_{k}^{i} \delta_{l}^{j+1} \mathbb{1}\right)_{(i, j) \in Z,(k, l) \in Z}, \\
& C_{1}=\left(\xi_{k, l}^{i+1, j}\right)_{(i, j) \in N^{c},(k, l) \in N^{c}}, \\
& C_{2}=\left(\xi_{k, l}^{i, j+1}\right)_{(i, j) \in N^{c},(k, i) \in N^{c}} .
\end{aligned}
$$

Here $\delta_{k}^{i}$ denotes Kronecker's delta. Furthermore, the converse is true:

Proposition 4. The above definition of $\xi$ gives a one-to-one correspondence between and

i) $w(\lambda) \in R\left[\left[\lambda_{1}^{-1}, \lambda_{2}^{-1}\right]\right]$ such that $w_{00}=1$,

ii) $\xi=\left(\xi_{k l}^{i j}\right)_{(i, j) \in Z,(k, l) \in N^{c}}, \xi_{k l}^{i j} \in R$, satisfying the following conditions:

$$
\begin{gathered}
\xi_{k l}^{i j}=\delta_{k}^{i} \delta_{l}^{j} \quad \text { if }(i, j) \in N^{c}, \\
\xi_{k l}^{i j}=0 \quad \text { if } i<k \geqq 0 \text { or } j<l \geqq 0 \\
\Lambda_{1} \xi=\xi C_{1}, \quad \Lambda_{2} \xi=\xi C_{2} \text { for some } N^{c} \times N^{c} \text {-matrices } C_{1}, C_{2} .
\end{gathered}
$$

Here the inverse correspondence $\xi \rightarrow w(\lambda)$ is defined by $w_{i j}=-\xi_{-i,-j}^{0,0}$.

Proof.

1) Proof of (2.1b). If $i<k$, then $w_{i-g, j-h}^{*} w_{g-k, h-l}=0$ for any $g, h \in \mathbb{Z}$ because $i-g<0$ or $g-k<0$ holds for any $g \in \mathbb{Z}$. If $j<l$, then $w_{i-g, j-h}^{*} w_{g-h, h-l}=0$ for any $g, h \in \mathbb{Z}$ because $j-h<0$ or $h-l<0$ holds for any $h \in \mathbb{Z}$. Therefore $\xi_{k l}^{i j}=\sum_{(g, h) \in N^{c}} w_{i-g, j-h}^{*} w_{g-k, h-l}=0$ if $i<k$ or $j<l$. 2) Proof of (2.1a). By the definition of $w_{i j}^{*}$, we obtain $\sum_{i+k=g, j+l=h} w_{i j}^{*} w_{k l}$
$=\delta_{0}^{g} \delta_{0}^{h}$. If $(i, j) \in N^{c}, i \geqq k$, and $j \geqq l$, then

$$
\begin{aligned}
\xi_{k l}^{i j} & =\sum_{(g, h) \in N^{c}} w_{i-g, j-h}^{*} w_{g-k, h-l} \\
& =\sum_{g=k}^{l} \sum_{h=l}^{j} w_{i-g, j-h}^{*} w_{g-k, h-l} \\
& =\sum_{g_{1}+g_{2}=i-k, h_{1}+h_{2}=j-l} w_{g_{1} h_{1}}^{*} w_{g_{2} h_{2}} \\
& =\delta_{0}^{i-k} \delta_{0}^{j-l} \mathbb{1} \\
& =\delta_{k}^{i} \delta_{l}^{j} \mathbb{1} .
\end{aligned}
$$

3) Proof of (2.1c). We denote $\Lambda=\left(\Lambda_{1}, \Lambda_{2}\right), \xi_{0}=\left(\delta_{k}^{i} \delta_{l}^{j} 1\right)_{(i, j) \in Z,(k, l) \in N^{c}}$, and $\Lambda_{a(-)}={ }^{t} \xi_{0} \Lambda_{a} \xi_{0}$ for $a=1,2$. Note that $\Lambda_{a} \xi_{0}=\xi_{0} \Lambda_{a(-)}$ for $a=1,2$, and

$$
\begin{aligned}
\xi & =\left(w_{i-k, j-l}^{*}\right)_{(i, j) \in Z .(k, l) \in N^{c}}\left(w_{i-k, j-l}\right)_{(i, j) \in N^{c},(k, l) \in N^{c}} \\
& =w(\Lambda)^{-1} \xi_{0}{ }^{t} \xi_{0} w(\Lambda) \xi_{0}=w(\Lambda)^{-1} \xi_{0} w(\Lambda)_{(-)},
\end{aligned}
$$


where we denote $w(\Lambda)_{(-)}={ }^{t} \xi_{0} w(\Lambda) \xi_{0}$. Then we obtain

$$
\begin{aligned}
\Lambda_{a} \xi & =\Lambda_{a} w(\Lambda)^{-1} \xi_{0} w(\Lambda)_{(-)}=w(\Lambda)^{-1} \Lambda_{a} \xi_{0} w(\Lambda)_{(-)} \\
& =w(\Lambda)^{-1} \xi_{0} \Lambda_{a(-)}{ }^{t} \xi_{0} w(\Lambda) \xi_{0}=w(\Lambda)^{-1} \xi_{0} w(\Lambda)_{(-)} C_{a}=\xi C_{a},
\end{aligned}
$$

where $C_{a}=\left\{w(\Lambda)_{(-)}\right\}^{-1} \Lambda_{a(-)} w(\Lambda)_{(-)}$.

4) Mapping (i) $\rightarrow$ (ii) $\rightarrow$ (i) is identity. In fact, if $w(\lambda) \rightarrow \xi \rightarrow \tilde{w}(\lambda)$, then

$$
\begin{aligned}
\tilde{w}_{i j} & =-\xi_{-i,-j}^{0.0} \\
& =-\sum_{(g . h) \in N^{c}} w_{-g,-h}^{*} w_{g+i, h+j} \\
& =\sum_{(g, h) \in N} w_{-g,-h}^{*} w_{g+i, h+j} \\
& =w_{i j} .
\end{aligned}
$$

5) Mapping (ii) $\rightarrow$ (i) $\rightarrow$ (ii) is identity. $\Lambda_{1} \xi=\xi C_{1}$ means $\xi_{k, l}^{i+1, j}=\sum_{(g, h) \in N^{c}} \xi_{g h}^{i j} C_{1 k l}^{g h}$, which reads $\xi_{k, l}^{i+1, j}=C_{1 k l}^{i j}$ when $(i, j) \in N^{c}$ because $\xi_{g h}^{i j}=\delta_{g}^{i} \delta_{h}^{j}$ for $(i, j) \in N^{c}$. Similarly, $\Lambda_{2} \xi=\xi C_{2}$ implies $C_{2 k l}^{i j}=\xi_{k, l}^{i, j+1}$ for $(i, j),(k, l) \in N^{c}$. Therefore, if $\Lambda_{1} \xi=\xi C_{1}$ and $\Lambda_{2} \xi$ $=\xi C_{2}$ for some $N^{c} \times N^{c}$-matrices $C_{1}, C_{2}$, then

$$
\begin{aligned}
& \xi_{k, l}^{i+1, j}=\sum_{(g, h) \in N^{c}} \xi_{g h}^{i j} \xi_{k, l}^{g+1, h}=\xi_{k-1, l}^{i, j}+\sum_{h=0}^{j} \xi_{-1, h}^{i, j} \xi_{k l}^{O h}, \\
& \xi_{k, l}^{i, j+1}=\sum_{(q, h) \in N^{c}} \xi_{g h}^{i j} \xi_{k, l}^{g, h+1}=\xi_{k, l-1}^{i, j}+\sum_{g=0}^{i} \xi_{k,-1}^{i, j} \xi_{k l}^{g 0} .
\end{aligned}
$$

This means that for any $m \in \mathbb{N},\left\{\xi_{k l}^{i j}\right\}(i, j) \in N,(k, l) \in N^{c}, i+j=m+1$ are determined by $\left\{\xi_{k l}^{l j}\right\}_{(i, j) \in N .(k, l) \in N^{c}, i+j \leqq m}$. Thus $\xi=\left(\xi_{k l}^{i j}\right)_{(i, j) \in Z,(k, l) \in N^{c}}$ is uniquely determined by $\left\{\tilde{\xi}_{k l}^{00}\right\}(k, l) \in N^{c}$, provided that $\xi$ satisfies $(2.1 \mathrm{a}),(2.1 \mathrm{~b})$, and $(2.1 \mathrm{c})$. Now we set $\xi \rightarrow w(\lambda) \rightarrow \widetilde{\xi}$. Then both $\xi$ and $\tilde{\xi}$ satisfy $(2.1 \mathrm{a}),(2.1 \mathrm{~b}),(2.1 \mathrm{c})$ and $\xi_{k l}^{00}=-w_{-k,-l}=\widetilde{\zeta}_{k l}^{00}$, from which $\xi=\tilde{\xi}$ follows. q.e.d.

Remark. The matrix $\xi$ can be regarded as an $N^{c}$-frame in the vector space $R^{Z}$, which represents a point in an infinite-dimensional Grassmann manifold. Then $\xi^{(+)}=\left(\xi_{k l}^{i j}\right)_{(l, j) \in N,(k, l) \in N^{c}}$ is regarded as a local coordinate system for the Grassmann manifold. Equations (2.1b) and (2.1c) are the defining equations for the relevant submanifold.

Now we rewrite Eqs. (1.9):

Theorem 1. Through the correspondence $w(\hat{\lambda}) \leftrightarrow \xi$, Eqs. (1.9) are equivalent to the existence of $N^{c} \times N^{c}$-matrices $B_{a b}(a, b=1,2)$ such that

$$
\left(-\Lambda_{a} \partial_{x_{a b}}+\partial_{t_{a b}}\right) \xi=\xi B_{a b} \quad(a, b=1,2) .
$$

Here $B_{a b}(a, b=1,2)$ are uniquely determined by $\xi$ if they exist, and (2.3) can be regarded as non-linear equations for $\xi$ as follows:

$$
\begin{aligned}
-\partial_{x_{1 b}} \xi_{k, l}^{i+1}+\partial_{t_{1 b}} \xi_{k l}^{i j} & =-\sum_{h \geqq 0} \xi_{-1, h}^{i, j} \partial_{x_{1 b}} \xi_{k l}^{0 h} \\
-\partial_{x_{2 b}} \xi_{k, j}^{i, j+1}+\partial_{t_{2 b}} \xi_{k l}^{i j} & =-\sum_{g \geqq 0} \xi_{g,-1}^{i, j} \partial_{x_{2 b}} \xi_{k l}^{00}(b=1,2) .
\end{aligned}
$$


Proof.

1) Proof that (1.9) implies (2.3).

$$
\begin{aligned}
\left(-\Lambda_{a} \partial_{x_{a b}}+\partial_{t_{a b}}\right) \xi= & \left(-\Lambda_{a} \partial_{x_{a b}}+\partial_{t_{a b}}\right)\left(w(\Lambda)^{-1} \xi_{0}{ }^{t} \xi_{0} w(\Lambda) \xi_{0}\right) \\
= & \left\{\left(-\Lambda_{a} \partial_{x_{a b}}+\partial_{t_{a b}}\right)\left(w(\Lambda)^{-1}\right)\right\} \xi_{0} w(\Lambda)_{(-)} \\
& +w(\Lambda)^{-1}\left(-\Lambda_{a} \partial_{x_{a b}}+\partial_{t_{a b}}\right) \xi_{0} w(\Lambda)_{(-)} \\
= & w(\Lambda)^{-1} A_{t_{a b}} \xi_{0} w(\Lambda)_{(-)}+w(\Lambda)^{-1}\left(-\Lambda_{a} \partial_{x_{a b}}+\hat{\partial}_{t_{a b}}\right) \xi_{0} w(\Lambda)_{(-)} \\
= & w(\Lambda)^{-1}\left(-\Lambda_{a} \partial_{x_{a b}}+\nabla_{t_{a b}}\right) \xi_{0} w(\Lambda)_{(-)} \\
= & w(\Lambda)^{-1} \xi_{0}\left(-\Lambda_{a(-)} \partial_{x_{a b}}+\nabla_{t_{a b}}\right) w(\Lambda)_{(-)} \\
= & w(\Lambda)^{-1} \xi_{0} w(\Lambda)_{(-)} B_{a b}=\xi B_{a b},
\end{aligned}
$$

where $B_{a b}=\left\{w(\Lambda)_{(-)}\right\}^{-1}\left(-\Lambda_{a(-)} \partial_{x_{a b}}+\nabla_{t_{a b}}\right) w(\Lambda)_{(-)}$, for any $a, b=1,2$.

2) Proof that (2.3) implies (2.4). In terms of entries, the equation $\left(-\Lambda_{1} \partial_{x_{1 b}}+\partial_{t_{1 b}}\right) \xi$ $=\xi B_{1 b}$ can be rewritten as

$$
-\partial_{x_{1 b}} \xi_{k, l}^{i+1, j}+\hat{\partial}_{t_{1 b}} \xi_{k l}^{i j}=\sum_{(g, h) \in N^{c}} \xi_{g h}^{i j} B_{k l}^{g h}
$$

which reads

$$
-\partial_{x_{1 b}} \xi_{k, l}^{i+1 . j}+\partial_{t_{1 b}} \xi_{k l}^{i j}=B_{k l}^{i j}
$$

when $(i, j) \in N^{c}$. Substituting this into $(2.5)$, we obtain

$$
\begin{aligned}
-\partial_{x_{1 b}} \xi_{k, l}^{i+1 . j}+\partial_{t_{1 b}} \xi_{k l}^{i j} & =\sum_{(g, h) \in N^{c}} \xi_{g h}^{l j}\left(-\partial_{x_{1 b}} \xi_{k, l}^{g+1, h}+\partial_{t_{1 b}} \xi_{k l}^{g h}\right) \\
& =-\sum_{h \geqq 0} \xi_{-1, h}^{i, j} \partial_{x_{1 b}} \xi_{k l}^{0 h}
\end{aligned}
$$

because $\xi_{k l}^{g h}=\dot{\delta}_{k}^{g} \delta_{l}^{h} \mathbb{1}$ for $(g, h) \in N^{c}$. The second equation of (2.4) can be derived in the same way.

3) Proof that (2.4) implies (1.9). When $i=j=0$, Eqs. (2.4) read

$$
\begin{aligned}
& -\partial_{x_{1 b}} \xi_{k l}^{10}+\partial_{t_{1 b}} \xi_{k l}^{00}=-\xi_{-1,0}^{0,0} \partial_{t_{1 b}} \xi_{k l}^{00}, \\
& -\partial_{x_{2 b}} \xi_{k l}^{01}+\partial_{t_{2 b}} \xi_{k l}^{00}=-\xi_{0,-1}^{0,0} \partial_{t_{2 b}} \xi_{k l}^{00} .
\end{aligned}
$$

Substituting $\xi_{k l}^{10}=-w_{1-k,-l}-w_{10}^{*} w_{-k,-l}, \quad \xi_{k l}^{00}=-w_{-k,-l}$, and $\xi_{k l}^{01}=-w_{-k, 1-l}$ $-w_{01}^{*} w_{-k,-l}$ into the above, we obtain

$$
\begin{aligned}
& -\partial_{x_{1 b}} w_{1-k,-l}+\partial_{t_{1 b}} w_{-k,-l}+\left(\partial_{x_{1 b}} w_{10}\right) w_{-k,-l}=0, \\
& -\partial_{x_{2 b}} w_{-k, 1-l}+\partial_{t_{2 b}} w_{-k,-l}+\left(\partial_{x_{2 b}} w_{01}\right) w_{-k,-l}=0,
\end{aligned}
$$

for any $(k, l) \in N^{c}$. Thus (2.4) implies all of Eqs. (1.9) except some trivial ones. q.e.d.

To investigate the structure of the solution space of (2.2), we consider an initialvalue problem with respect to the subspace $t=0$. Unlike the case of self-dual YangMills equations, we cannot solve it for arbitrary data; the data for which it is 
solvable must satisfy a system of differential equations. In fact, we have

Proposition 5. The system of equations (2.1a), (2.1b), (2.1c), and (2.3) implies that

if $k \geqq 0$ and $p+q>i-k$, then $\partial_{x_{11}}^{p} \partial_{x_{12}}^{q} \xi_{k l}^{i j}=0 \quad\left((i, j) \in Z,(k, l) \in N^{c}\right)$,

if $l \geqq 0$ and $p+q>j-l$, then $\partial_{x_{21}}^{p} \partial_{x_{22}}^{q} \xi_{k l}^{i j}=0\left((i, j) \in Z,(k, l) \in N^{c}\right)$.

Under the conditions (2.1a), (2.1b), and (2.1c), Eqs. (2.6) are equivalent to the following equations:

and

$$
\text { If } p+q=i+1, \text { then } \partial_{x_{11}}^{p} \partial_{x_{12}}^{q} \xi_{0 l}^{i 0}=0, \quad(p, q, i \geqq 0, l<0)
$$

$$
\text { if } p+q=j+1, \quad \text { then } \partial_{x_{21}}^{p} \partial_{x_{22}}^{q} \xi_{k 0}^{0 j}=0, \quad(p, q, j \geqq 0, k<0)
$$

Proof.

1) It is obvious that (2.6) implies (2.7)

2) Proof that (2.4) implies (2.6). It follows from (2.1b) that $\xi_{k l}^{0 h}=0$ for $k>0$. Thus the first equation of (2.4) reads

$$
-\partial_{x_{1 b}} \xi_{k, l}^{i+1, j}+\hat{o}_{t_{1 b}} \xi_{k l}^{i j}=0
$$

for $k>0$. Thanks to this formula, the first equation of (2.6) can be proved by induction starting from the case $i-k=-1$ which is trivial. We cannot use the formula when $k=0$, but Eqs. (2.6) also hold for $k=0$ because $\xi_{k l}^{i j}=\xi_{0, l}^{i-k, j}$ for any $k \geqq 0$. The second equation of (2.6) can be derived similarly.

3) Proof that (2.7) implies (2.6). We shall prove the first equation only. (The second one can be proved similarly.) Since $\xi_{k l}^{i j}=\xi_{0, l}^{i-k, j}$ for $k \geqq 0$, it is sufficient to show that

$$
\text { if } p+q>i \text {, then } \partial_{x_{11}}^{p} \partial_{x_{12}}^{q} \xi_{0 l}^{i j}=0 \text {. }
$$

We shall prove this by induction on $j$. The case $j=0$ is just (2.7). Assume that (2.9) holds for any $j \leqq m$. When $k=0$, the second equation of (2.2) reads

$$
\xi_{0, l}^{i, m+1}=\xi_{0, l-1}^{i, m}+\sum_{g=0}^{i} \xi_{g,-1}^{i, m} \xi_{0 l}^{g 0}=\xi_{0, l-1}^{i, m}+\sum_{g=0}^{i} \xi_{0,-1}^{i-g, m} \xi_{0 l}^{g 0} .
$$

Differentiating both sides, we obtain

$$
\begin{aligned}
\partial_{x_{11}}^{p} \partial_{x_{12}}^{q} \xi_{0, l}^{i, m+1}= & \partial_{x_{11}}^{p} \partial_{x_{12}}^{q} \xi_{0, l-1}^{i, m} \\
& +\sum_{g=0}^{i} \sum_{r=0}^{p} \sum_{s=0}^{q}\left(\begin{array}{l}
p \\
r
\end{array}\right)\left(\begin{array}{l}
q \\
s
\end{array}\right) \partial_{x_{11}}^{p-r} \partial_{x_{12}}^{q-s} \xi_{0,-1}^{i-g, m} \partial_{x_{11}}^{r} \partial_{x_{12}}^{s} \xi_{0 l}^{g 0} .
\end{aligned}
$$

If $p+q>i$, then $\partial_{x_{11}}^{p} \partial_{x_{12}}^{q} \xi_{0, l-1}^{i . m}=0$ and either $\partial_{x_{11}}^{p-r} \partial_{x_{12}}^{q-s} \xi_{0,-1}^{i-g, m}=0$ or $\partial_{x_{11}}^{r} \partial_{x_{12}}^{s} \xi_{0 l}^{g 0}=0$ holds by the assumption of induction. Thus (2.9) holds for $j=m+1$. q.e.d.

Conversely, for any initial datum satisfying (2.6) (or (2.7)) we can solve the initial-value problem: 
Theorem 2. For any $\xi^{(0)}=\left(\xi_{k l}^{(0) i j}\right)_{(i, j) \in Z,(k, l) \in N^{c}}, \xi_{k l}^{(0) i j} \in M_{n}(\mathbb{C})[[x]]$ satisfying $(2.1 \mathrm{a})$, (2.1b), (2.1c), and (2.6) (or (2.7)), there exists a unique solution $\xi$ to the initial-value problem, i.e. $\xi=\left(\xi_{k l}^{i j}\right)_{(i, j) \in Z,(k, l) \in N^{c}}, \xi_{k l}^{i j} \in M_{n}(\mathbb{C})[[t, x]]$ satisfying $(2.1 \mathrm{a}),(2.1 \mathrm{~b}),(2.1 \mathrm{c})$, (2.3), and $\left.\xi\right|_{t=0}=\xi^{(0)}$. The solution $\xi$ has the following form:

where

$$
\xi=\tilde{\xi}\left(\xi^{(-)}\right)^{-1}
$$

$$
\begin{gathered}
\tilde{\xi}=\exp \left(\sum_{a=1}^{2} \sum_{b=1}^{2} t_{a b} \Lambda_{a} \partial_{x_{a b}}\right) \xi^{(0)}, \\
\xi=\left(\begin{array}{c}
\tilde{\xi}^{(-)} \\
\tilde{\xi}^{(+)}
\end{array}\right), \quad \tilde{\xi}^{(-)}=\left(\tilde{\xi}_{k l}^{i j}\right)_{(i, j) \in N^{c},(k, l) \in N^{c}}, \\
\tilde{\xi}^{(+)}=\left(\widetilde{\xi}_{k l}^{i j}\right)_{(i, j) \in N,(k, l) \in N^{c}} .
\end{gathered}
$$

Proof.

Proof of the uniqueness. Set

and

$$
w(\Lambda)=\sum_{i, j, k, l \geqq 0} w_{i j k l}(\lambda) t_{11}^{l} t_{12}^{j} t_{21}^{k} t_{22}^{l}
$$

$$
w_{i j k l}(\hat{\lambda})=\sum_{g, h \geqq 0} w_{g h ; i j k l} \lambda_{1}^{-g} \lambda_{2}^{-h}
$$

Then (1.9) are recursion formulae for $w_{i j k l}(\lambda)$ :

$$
\begin{aligned}
(i+1) w_{i+1, j . k, l}(\lambda) & =\lambda_{1} \partial_{x_{11}} w_{i j k l}(\lambda) \\
& -\sum_{p=0}^{i} \sum_{q=0}^{j} \sum_{r=0}^{k} \sum_{s=0}^{l}\left(\partial_{x_{11}} w_{10 ; i-p, j-q . k-r, l-s}\right) w_{p q r s}(\lambda)
\end{aligned}
$$

etc. Thus $\left\{w_{i j k l}(\hat{\lambda})\right\}_{i, j, k . l \geqq 0}$ are uniquely determined by $w_{0000}(\lambda)$ if they exist. This completes the proof because of the one-to-one correspondence $w(\lambda) \leftrightarrow \xi$ in Proposition 4.

\section{Proof of the solution formulae}

1) Let

$$
\begin{aligned}
\mathscr{R}= & \left\{A=\left(A_{k l}^{i j}\right)_{(i, j) \in N^{c},(k, l) \in N^{c}} \mid A_{k l}^{i j} \in M_{n}(\mathbb{C})[[x]], \text { there exists an integer } m \text { such that } A_{k l}^{i j}\right. \\
& =0 \text { if } i-k \leqq m \text { or } j-l \leqq m\}, \\
\mathscr{F}= & \left\{\xi=\left(\xi_{k l}^{i j}\right)_{(i, j) \in Z,(k, l) \in N^{c}} \mid \xi_{k l}^{i j} \in M_{n}(\mathbb{C})[[x]], \text { there exists an integer } m \text { such that } \xi_{k l}^{i j}\right. \\
& =0 \text { if } i-k \leqq m \text { or } j-l \leqq m\},
\end{aligned}
$$

and $\mathscr{F}[[t]]=\left\{\sum_{i \geqq 0} \xi_{i} t^{i} \mid \xi_{i} \in \mathscr{F}\right\}$.

$\mathscr{R}$ is a $\mathbb{C}$-algebra on which $\Lambda_{a(-)} \in \mathscr{R}(a=1,2)$ and $\partial_{x_{a b}}(a, b=1,2)$ act. $\mathscr{F}$ is a right $\mathscr{R}$-module with $\mathscr{R}$-action defined by multiplication as matrices. $\Lambda_{a}(a=1,2)$ and 
$\partial_{x_{a b}}(a, b=1,2)$ act on $\mathscr{F}$ from the left. Since $\xi^{(0)} \in \mathscr{F}$,

$$
\tilde{\xi}=\sum_{p \geqq 0} \frac{1}{p !}\left(\sum_{a=1}^{2} \sum_{b=1}^{2} t_{a b} \Lambda_{a} \partial_{x_{a b}}\right)^{p} \xi^{(0)}
$$

is well-defined as an element of $\mathscr{F}[[t]]$.

The following proposition is important because it means that the system of Eqs. (2.1c) and (2.3) defines a motion on an infinite-dimensional Grassmann manifold:

Proposition 6. The system of equations (2.1c) and (2.3) is invariant under change of frame: let $\xi \in \mathscr{F}[[t]]$ and $C_{a}, B_{a b} \in \mathscr{R}[[t]](a, b=1.2)$ satisfy (2.1c) and (2.3). For any invertible element $P \in \mathscr{R}[[t]]$, set $\xi^{\prime}=\xi P, C_{a}^{\prime}=P^{-1} C_{a} P$, and $B_{a b}^{\prime}=P^{-1}\left(B_{a b}-C_{a} \partial_{x_{a b}}\right.$ $\left.+\partial_{t_{a b}}\right)$ P for $a, b=1,2$. Then $\xi^{\prime}, C_{a}^{\prime}$, and $B_{a b}^{\prime}(a, b=1,2)$ also satisfy $(2.1 \mathrm{c})$ and $(2.3)$.

Proof. The following calculation proves the proposition:

$$
\begin{aligned}
\Lambda_{a} \xi^{\prime}=\Lambda_{a} \xi P & =\xi C_{a} P=\xi P P^{-1} C_{a} P=\xi^{\prime} C_{a}^{\prime}(a=1,2) \\
\left(-\Lambda_{a} \partial_{x_{a b}}+\hat{\partial}_{t_{a b}}\right) \xi^{\prime} & =\left(-\Lambda_{a} \partial_{x_{a b}}+\partial_{t_{a b}}\right)(\xi P) \\
& =\left(\left(-\Lambda_{a} \partial_{x_{a b}}+\partial_{t_{a b}}\right) \xi\right) P-\Lambda_{a} \xi \partial_{x_{a b}} P+\xi \partial_{t_{a b}} P \\
& =\xi B_{a b} P-\xi C_{a} \partial_{x_{a b}} P+\xi \hat{\partial}_{t_{a b}} P \\
& =\xi\left(B_{a b}-C_{a} \partial_{x_{a b}}+\partial_{t_{a b}}\right) P \\
& =\xi P P^{-1}\left(B_{a b}-C_{a} \partial_{x_{a b}}+\partial_{t_{a b}}\right) P \\
& =\xi^{\prime} B^{\prime}{ }_{a b}(a, b=1,2) . \text { q.e.d. }
\end{aligned}
$$

2) In terms of entries, the definition of $\widetilde{\xi}$ can be written in the following form:

$$
\xi_{k l}^{i j}=\sum_{p, q \geqq 0} \frac{1}{p !}\left(t_{11} \partial_{x_{11}}+t_{12} \partial_{x_{12}}\right)^{p} \frac{1}{q !}\left(t_{21} \partial_{x_{21}}+t_{22} \partial_{x_{22}}\right)^{q} \xi_{k, l}^{(0) i+p, j+q} .
$$

Since $\xi^{(0)}$ satisfies (2.6), we obtain

$$
\tilde{\xi}_{k l}^{i j}=0 \quad \text { if } \quad i<k \geqq 0 \text { or } \quad j<l \geqq 0
$$

3) It follows immediately from the definition of $\tilde{\xi}$ that

$$
\left(-\Lambda_{a} \partial_{x_{a b}}+\partial_{t_{a b}}\right) \widetilde{\xi}=0 \quad(a, b=1,2) .
$$

4) There exist $\widetilde{C}_{1}, \widetilde{C}_{2} \in \mathscr{R}[[t]]$ such that

$$
\Lambda_{a} \widetilde{\xi}=\widetilde{\xi} \widetilde{C}_{a}(a=1,2) .
$$

This can be proved as follows: let $w^{(0)}(\lambda) \leftrightarrow \xi^{(0)}$ through the correspondence in Proposition 4. Then $\xi^{(0)}=w^{(0)}(\Lambda)^{-1} \xi_{0} w^{(0)}(\Lambda)_{(-)}$, where $w^{(0)}(\Lambda)_{(-)}$ $={ }^{t} \xi_{0} w^{(0)}(\Lambda) \xi_{0}$. Set $\Lambda_{(-)}=\left(\Lambda_{1(-)}, \Lambda_{2(-)}\right), P(\Lambda)=\sum_{a=1}^{2} \sum_{b=1}^{2} t_{a b} \Lambda_{a} \partial_{x_{a b}}$, and $P\left(\Lambda_{(-)}\right)$ $=\sum_{a=1}^{2} \sum_{b=1}^{2} t_{a b} \Lambda_{a(-)} \hat{o}_{x_{a b}}$. We note that $\Lambda_{a} \xi_{0}=\xi_{0} \Lambda_{a(-)}$ for $a=1,2$ and hence that 
$P(\Lambda) \xi_{0}=\xi_{0} P\left(\Lambda_{(-)}\right)$. Then it follows from the definition of $\xi$ that

$$
\begin{aligned}
\xi & =\sum_{i \geqq 0} \frac{1}{i !} P(\Lambda)^{i} w^{(0)}(\Lambda)^{-1} \xi_{0} w^{(0)}(\Lambda)_{(-)} \\
& =\sum_{i \geqq 0} \sum_{j \geqq 0} \frac{1}{(i-j) !} P(\Lambda)^{i-j} w^{(0)}(\Lambda)^{-1} \frac{1}{j !} P(\Lambda)^{j} \xi_{0} w^{(0)}(\Lambda)_{(-)} \\
& =\sum_{k \geqq 0} \frac{1}{k !} P(\Lambda)^{k} w^{(0)}(\Lambda)^{-1} \xi_{0} \sum_{j \geqq 0} \frac{1}{j !} P\left(\Lambda_{(-)}\right)^{j} w^{(0)}(\Lambda)_{(-)} \\
& =\exp [P(\Lambda)] w^{(0)}(\Lambda)^{-1} \xi_{0} \exp \left[P\left(\Lambda_{(-)}\right)\right] w^{(0)}(\Lambda)_{(-)},
\end{aligned}
$$

and that

$$
\begin{aligned}
\Lambda_{a} \tilde{\xi} & =\Lambda_{a} \exp [P(\Lambda)] w^{(0)}(\Lambda)^{-1} \xi_{0} \exp \left[P\left(\Lambda_{(-)}\right)\right] w^{(0)}(\Lambda)_{(-)} \\
& =\exp [P(\Lambda)] w^{(0)}(\Lambda)^{-1} \xi_{0} \Lambda_{a(-)} \exp \left[P\left(\Lambda_{(-)}\right)\right] w^{(0)}(\Lambda)_{(-)} \\
& =\widetilde{\xi} \tilde{C}_{a},
\end{aligned}
$$

where $\tilde{C}_{a}=\left\{\exp \left[P\left(\Lambda_{(-)}\right)\right] w^{(0)}(\Lambda)_{(-)}\right\}^{-1} \Lambda_{a(-)} \exp \left[P\left(\Lambda_{(-)}\right)\right] w^{(0)}(\Lambda)_{(-)}$. The invertibility of $\exp \left[P\left(\Lambda_{(-)}\right)\right] w^{(0)}(\Lambda)_{(-)}$in $\mathscr{R}[[t]]$ follows from the fact that $\left.\exp \left[P\left(\Lambda_{(-)}\right)\right] w^{(0)}(\Lambda)_{(-)}\right|_{t=0}=w^{(0)}(\Lambda)_{(-)}$and that $w^{(0)}(\Lambda)_{(-)}$ $=\left(w_{i-k, j-l}^{(0)}\right)_{(i, j) \in N^{c},(k, l) \in N^{c}}$ is invertible in $\mathscr{R}$.

5) It follows from (2.13) and (2.14) that $\tilde{\xi}$ is a solution of the system of (2.1c) and (2.3) for $B_{a b}=0(a, b=1,2)$. $\widetilde{\xi}_{(-)} \in \mathscr{R}[[t]]$ follows from that $\widetilde{\xi}_{\in} \mathscr{F}[[t]]$, and $\widetilde{\xi}_{(-)}$is invertible in $\mathscr{R}[[t]]$ because $\left.\widetilde{\xi}_{(-)}\right|_{t=0}=\left(\delta_{k}^{i} \delta_{l}^{j} \mathfrak{l}\right)_{(i, j) \in N^{c},(k, l) \in N^{c}}=1 \in \mathscr{R}$. Then Proposition 6 says that $\xi=\widetilde{\xi}\left(\widetilde{\xi}_{(-)}\right)^{-1} \in \mathscr{F}[[t]]$ satisfies (2.1c) and (2.3). Equation (2.1a) follows from the definition of $\xi$. Thus the last to prove is $(2.1 \mathrm{~b})$. Let

and

$$
\begin{array}{lll}
\mathscr{R}_{1}=\left\{A \in \mathscr{R} \mid A_{k l}^{i j}=0\right. & \text { if } \quad i<k \geqq 0 & \text { or } \quad j<l \geqq 0\}, \\
\mathscr{F}_{1}=\left\{\xi \in \mathscr{F} \mid \xi_{k l}^{i j}=0\right. & \text { if } \quad i<k \geqq 0 & \text { or } \quad j<l \geqq 0\},
\end{array}
$$

$$
\mathscr{F}_{1}[[t]]=\left\{\sum_{i \geqq 0} \xi_{i} t^{i} \mid \xi_{i} \in \mathscr{F}_{1}\right\} .
$$

Then $\mathscr{R}_{1}$ is a subring of $\mathscr{R}$ and $\mathscr{F}_{1}$ is an $\mathscr{R}_{1}$-module. $\tilde{\xi}$ is an element of $\mathscr{F}_{1}[[t]]$ and $\tilde{\xi}_{(-)}$is an invertible element of $\mathscr{R}_{1}[[t]]$ because of (2.12). Therefore $\xi=\tilde{\xi}\left(\widetilde{\xi}_{(-)}\right)^{-1} \in \mathscr{F}_{1}[[t]]$. This completes the proof of Theorem 2 .

In summary, by choosing the proper frame, the time evolutions in the initialvalue problem can be regarded as evolutions defined by linear differential equations, and the solution space of (1.9) is faithfully parametrized by the solution space of Eqs. (2.6)[or (2.7)] in the subspace $t=0$.

\section{Relation to the Yang-Mills Fields}

First we describe the procedure for obtaining Yang-Mills potentials from any solution of Eqs. (1.9) (or (2.4)): 
Proposition 7. Given any solution of Eqs. (1.9) (or (2.4)), set

$$
\begin{aligned}
& A_{x_{0}}=-\left(A_{t_{12}}+A_{t_{22}}\right)=-\left(\partial_{x_{12}} w_{10}+\partial_{x_{22}} w_{01}\right)=\partial_{x_{12}} \xi_{-1,0}^{00}+\partial_{x_{22}} \xi_{0,-1}^{00}, \\
& A_{x_{1}}=\sqrt{-1}\left(A_{t_{12}}+A_{t_{22}}\right)=\sqrt{-1}\left(\partial_{x_{12}} w_{10}+\partial_{x_{22}} w_{01}\right) \\
& =-\sqrt{-1}\left(\partial_{x_{12}} \xi_{-1,0}^{00}+\hat{\partial}_{x_{22}} \xi_{0,-1}^{00}\right), \\
& A_{x_{2}}=A_{t_{11}}+A_{t_{21}}=\hat{O}_{x_{11}} w_{10}+\hat{\partial}_{x 21} w_{01}=-\left(\partial_{x_{11}} \xi_{-1,0}^{00}+\partial_{x_{21}} \xi_{0,-1}^{00}\right)
\end{aligned}
$$

$$
\begin{aligned}
A_{x_{3}} & =\sqrt{-1}\left(A_{t_{11}}-A_{t_{21}}\right)=\sqrt{-1}\left(\partial_{x_{11}} w_{10}+\partial_{x_{21}} w_{01}\right) \\
& =-\sqrt{-1}\left(\partial_{x_{11}} \xi_{-1,0}^{00}+\partial_{x_{21}} \xi_{0 .-1}^{00}\right) .
\end{aligned}
$$

Substitute

$$
\begin{aligned}
& x_{11}=x_{21}=x_{0}+\sqrt{-1} x_{1}, t_{11}=t_{22}=x_{2}+\sqrt{-1} x_{3}, \\
& i_{12}=t_{22}=-x_{0}+\sqrt{-1} x_{1}, x_{12}=t_{21}=x_{2}-\sqrt{-1} x_{3}
\end{aligned}
$$

into the above. Then $A=\left(A_{x_{0}}, A_{x_{1}}, A_{x_{2}}, A_{x_{3}}\right)$ gives a set of Yang-Mills potentials (i.e. a solution of the system $(0.2))$.

Proposition 8. Let $\nabla$ and $\nabla^{\prime}$ be gauge fields in $\mathbb{C}^{8}$ satisfying (0.1). If $\nabla$ and $\nabla^{\prime}$ are gauge-equivalent as Yang-Mills fields in the diagonal subspace 4 , then they are gaugeequivalent in $\mathbb{C}^{8}$.

Proof. If $\nabla$ and $\nabla^{\prime}$ are gauge-equivalent as gauge fields on $\Delta$, there exists $g=g(x) \in M_{n}(\mathbb{C})\left[\left[x_{0}, x_{1}, x_{2}, x_{3}\right]\right]$ such that $\nabla_{x_{\mu}}=g^{-1} \nabla_{x_{\mu}}^{\prime} g$ on $\Delta$ for $\mu=0,1,2,3$. Set $\widetilde{\nabla}=g^{-1} \nabla^{\prime} g$ in $\mathbb{C}^{8}$. Then $\widetilde{\nabla}$ is gauge-equivalent to $\nabla^{\prime}$ by definition and $\tilde{\nabla}_{x_{\mu}}=\nabla_{x_{\mu}}$ on $\Delta$ for $\mu=0,1,2,3$. It is sufficient to prove that $\tilde{\nabla}$ and $\nabla$ are gauge-equivalent.

First we note that Eqs. (0.1) are rewritten in terms of $\nabla_{x_{\mu}}$ and $\nabla_{w_{\mu}}$ as follows:

$$
\begin{aligned}
& {\left[\nabla_{w_{\mu}}, \nabla_{x_{\nu}}\right]=\sum_{\kappa=0}^{3} \sum_{\lambda=0}^{3}(1 / 2) \varepsilon_{\mu v \kappa \lambda}\left[\nabla_{x_{\kappa}}, \nabla_{x_{\lambda}}\right],} \\
& {\left[\nabla_{w_{\mu}}, \nabla_{w_{v}}\right]=\left[\nabla_{x_{\mu}}, \nabla_{x_{v}}\right](\mu, v=0,1,2,3) .}
\end{aligned}
$$

Expanding $\nabla$ with respect to $w$ as

$$
\begin{array}{ll}
\nabla_{x_{\mu}}=\partial_{x_{\mu}}+\sum_{\alpha \in \mathbb{N}^{4}} A_{x_{\mu}}^{\alpha} w^{\alpha}, & A_{x_{\mu}}^{\alpha}=A_{x_{\mu}}^{\alpha}(x) \in M_{n}(\mathbb{C})\left[\left[x_{0}, x_{1}, x_{2}, x_{3}\right]\right], \\
\nabla_{w_{\mu}}=\hat{o}_{w_{\mu}}+\sum_{\gamma \in \mathbb{N}^{4}} A_{w_{\mu}}^{\alpha} w^{\alpha}, & A_{w_{\mu}}^{\alpha}=A_{w_{\mu}}^{\alpha}(x) \in M_{n}(\mathbb{C})\left[\left[x_{0}, x_{1}, x_{2}, x_{3}\right]\right],
\end{array}
$$

and substituting this into (2.1), we obtain

$$
\begin{aligned}
\left(\alpha_{\mu}+1\right) A_{\lambda_{\nu}}^{\alpha+e_{\mu}=}= & \hat{\partial}_{x_{v}} A_{w_{\mu}}^{\alpha}-\sum_{\beta}\left[A_{w_{\mu}}^{\alpha-\beta}, A_{x_{\nu}}^{\beta}\right] \\
& +\sum_{\kappa=0}^{3} \sum_{\lambda=0}^{3}(1 / 2) \varepsilon_{\mu \nu \kappa \lambda}\left(\hat{\partial}_{x_{\kappa}} A_{x_{\lambda}}^{\alpha}-\partial_{x_{\lambda}} A_{x_{\kappa}}^{\alpha}+\sum_{\beta}\left[A_{x_{\kappa}}^{\alpha-\beta}, A_{x_{\lambda}}^{\beta}\right]\right),
\end{aligned}
$$




$$
\begin{aligned}
\left(\alpha_{\mu}+1\right) A_{w_{v}}^{\alpha+e_{\mu}}\left(\alpha_{v}+1\right) A_{w_{\mu}}^{\alpha+e_{v}}= & -\sum_{\beta}\left[A_{w_{\mu}}^{\alpha-\beta}, A_{w_{v}}^{\beta}\right] \\
& \left.+\partial_{x_{\mu}} A_{x_{v}}^{\alpha}-\partial_{x_{v}} A_{x_{\mu}}^{\alpha}+\sum_{\beta}\left[A_{x_{\mu}}^{\alpha-\beta}, A_{x_{v}}^{\beta}\right]\right)
\end{aligned}
$$

where $e_{0}=(1,0,0,0), e_{1}=(0,1,0,0), e_{2}=(0,0,1,0), e_{3}=(0,0,0,1) \in \mathbb{N}^{4}$. We may assume without loss of generality that $\sum_{\mu=0}^{3} A_{w_{\mu}}^{\alpha-e_{\mu}}=0$ for any $\alpha \in \mathbb{N}^{4}$ by virtue of gauge transformation. Then $\left\{A_{w_{\mu}}^{\alpha}\right\}_{\alpha \in \mathbb{N}^{4}, \mu=0,1,2,3}$ and $\left\{A_{x_{\mu}}^{\alpha}\right\}_{\alpha \in \mathbb{N}^{4}, \mu=0,1,2,3}$ are recursively and uniquely determined by $\left\{A_{x_{\mu}}^{0}\right\}_{\mu=0,1,2,3}$. Finally, we prove the existence of such gauge transformation. Let $m$ be any positive integer and let $g_{m}=\mathbb{1}-\sum_{|\alpha|=m} g_{m}^{\alpha} w^{\alpha}$. Then $g_{m}^{-1}=\sum_{j \geqq 0}\left(\sum_{|\alpha|=m} g_{m}^{\alpha} w^{\alpha}\right)^{j}$. Let $A_{w_{\mu}} \rightarrow \tilde{A}_{w_{\mu}}=g_{m}^{-1} A_{w_{\mu}} g_{m}$ $+g_{m}^{-1}\left(\hat{o}_{w_{\mu}} g_{m}\right)$. Then $\tilde{A}_{w_{\mu}}^{\alpha}=A_{w_{\mu}}^{\alpha}$ if $|\alpha|<m-1, \tilde{A}_{w_{\mu}}^{\alpha}=A_{w_{\mu}}^{\alpha}-\left(\alpha_{\mu}+1\right) g_{m}^{\alpha+e_{\mu}}$ if $|\alpha|=m-1$, and hence $\sum_{\mu=0}^{3} \tilde{A}_{w_{\mu}}^{\alpha-e_{\mu}}=\sum_{\mu=0}^{3} A_{w_{\mu}}^{\alpha-e_{\mu}}-m g_{m}^{\alpha}$ if $|\alpha|=m$. Thus for any given $A$, we define $\left\{g_{m}=\mathbb{1}-\sum_{|\alpha|=m} g_{m}^{\alpha} w^{\alpha}\right\}_{m \geqq 1}$ inductively by

$$
\begin{array}{ll}
g_{1}^{\alpha}=\sum_{\mu=0}^{3} A_{w_{\mu}}^{\alpha-e_{\mu}}, & A_{1, w_{\mu}}=g_{1}^{-1} A_{w_{\mu}} g_{1}+g_{1}^{-1}\left(\partial_{w_{\mu}} g_{1}\right), \\
g_{m}^{\alpha}=\sum_{\mu=0}^{3} A_{m-1, w_{\mu}}^{\alpha-e_{\mu}}, & A_{m, w_{\mu}}=g_{m}^{-1} A_{m-1, w_{\mu}} g_{m}+g_{m}^{-1}\left(\partial_{w_{\mu}} g_{m}\right)
\end{array}
$$

and set $g=\prod_{j \geqq 1} g_{j}=g_{1} g_{2} g_{3} \ldots$ q.e.d.

For any self-dual Yang-Mills field $\nabla$, i.e. covariant derivatives $\nabla_{x_{\mu}}=\partial_{x_{\mu}}$ $+A_{x_{\mu}}(x)$ satisfying

$$
\left[\nabla_{x_{\mu}}, \nabla_{x_{v}}\right]=(1 / 2) \sum_{\alpha=0}^{3} \sum_{\beta=0}^{3} \varepsilon_{\mu \nu \alpha \beta}\left[\nabla_{x_{\alpha}}, \nabla_{x_{\beta}}\right](\mu, v=0,1,2,3),
$$

define a gauge field $\tilde{\nabla}$ on $\mathbb{C}^{8}$ by

$$
\begin{array}{ll}
\tilde{\nabla}_{y_{\mu}}=\partial_{y_{\mu}}+\tilde{A}_{y_{\mu}}, & \tilde{A}_{y_{\mu}}=A_{x_{\mu}}(y), \\
\tilde{\nabla}_{z_{\mu}}=\hat{C}_{z_{\mu}}+\tilde{A}_{z_{\mu}}, & \tilde{A}_{z_{\mu}}=0(\mu=0,1,2,3) .
\end{array}
$$

Then we obtain

$$
\begin{aligned}
& {\left[\tilde{\nabla}_{y_{\mu}}, \tilde{\nabla}_{y_{v}}\right]=(1 / 2) \sum_{\alpha=0}^{3} \sum_{\beta=0}^{3} \varepsilon_{\mu v \alpha \beta}\left[\tilde{\nabla}_{y_{\alpha}}, \tilde{\nabla}_{y_{\beta}}\right],} \\
& {\left[\tilde{\nabla}_{z_{\mu}}, \tilde{\nabla}_{z_{v}}\right]=0,} \\
& {\left[\tilde{\nabla}_{y_{\mu}}, \tilde{\nabla}_{z_{v}}\right]=0 \quad(\mu, v=0,1,2,3),}
\end{aligned}
$$

which imply Eqs. (0.1). Thus all the self-dual fields belong to the class of YangMills fields given by the restriction of Witten's gauge fields (0.1). Note that the trivial extension (3.2) is the unique one up to gauge equivalence by virtue of 
Proposition 8 and that Eqs. (3.3) are gauge-invariant. Therefore, if any gauge field $\nabla$ satisfies $(0.1)$ and its restriction to the diagonal subspace $\Delta$ is self-dual, then $\nabla$ satisfies (3.3). Conversely, suppose that a gauge field $\widetilde{\nabla}$ satisfies (3.3). We may assume that $\tilde{A}_{z_{\mu}}=0$, because such a gauge can be taken by virtue of the equations $\left[\widetilde{\nabla}_{z_{\mu}}, \tilde{\nabla}_{z_{v}}\right]=0(\mu, v=0,1,2,3)$. Then $0=\left[\tilde{\nabla}_{y_{\mu}}, \tilde{\nabla}_{z_{v}}\right]=\left[\tilde{\nabla}_{y_{\mu}}, \partial_{z_{v}}\right]=-\partial_{z_{v}} A_{y_{\mu}}$, i.e., $\tilde{A}_{y_{\mu}}=\widetilde{A}_{y_{\mu}}(y)$. Set $A_{x_{\mu}}=\tilde{A}_{y_{\mu}}(x)$ and $\nabla_{x_{\mu}}=\partial_{x_{\mu}}+A_{x_{\mu}}$. Then we obtain $\left[\nabla_{x_{\mu}}, \nabla_{x_{v}}\right]$ $=(1 / 2) \sum_{\alpha=0}^{3} \sum_{\beta=0}^{3} \varepsilon_{\mu v \alpha \beta}\left[\nabla_{x_{\alpha}}, \nabla_{x_{\beta}}\right]$ and $\nabla_{x_{\mu}}=\left.\widetilde{\nabla}_{x_{\mu}}\right|_{w=0}$. Thus we have

Proposition 9. The solutions $\nabla$ of (0.1) which correspond to self-dual or anti-self-dual fields on $\triangle$ are characterized by

or

$$
\left[\nabla_{z_{\mu}}, \nabla_{z_{v}}\right]=0(\mu, v=0,1,2,3)
$$

$$
\left[\nabla_{y_{\mu}}, \nabla_{y_{v}}\right]=0(\mu, v=0,1,2,3)
$$

respectively. All the self-dual or anti-self-dual Yang-Mills fields can be obtained in this way.

Rewriting (3.4) in terms of $\xi$, we obtain

Proposition 10. (i) A solution $\xi$ to the system of Eqs. (2.1a), (2.1b), (2.1c), and (2.3) corresponds to a self-dual field on $\Delta$ if and only if it satisfies

$$
\partial_{x_{21}}^{2} \xi_{0,-1}^{0,0}=\partial_{x_{21}} \partial_{x_{22}} \xi_{0,-1}^{0,0}=\partial_{x_{22}}^{2} \xi_{0,-1}^{0,0}=0 \text {. }
$$

(ii) A solution $\xi$ to the system of Eqs. (2.1a), (2.1b), (2.1c), and (2.3) corresponds to an anti-self-dual field on $\Delta$ if and only if it satisfies

$$
\partial_{x_{11}}^{2} \xi_{-1,0}^{0,0}=\partial_{x_{11}} \partial_{x_{12}} \xi_{-1,0}^{0,0}=\partial_{x_{12}}^{2} \xi_{-1,0}^{0,0}=0
$$

Proof. We prove (i) only. Noting that $\left(x_{21}, x_{22}, t_{21}, t_{22}\right)$ are the coordinates of $z$-space, we can see that (3.4), the integrability in $z$-directions is equivalent to the following system:

$$
\begin{aligned}
& {\left[\nabla_{x_{21}}, \nabla_{x_{22}}\right]=\left[\nabla_{t_{21}}, \nabla_{t_{22}}\right]=0,} \\
& {\left[\nabla_{x_{2 a}}, \nabla_{t_{2 b}}\right]=0(a, b=1,2) .}
\end{aligned}
$$

The first two equations are trivial since we assume that the gauge field satisfies (1.1). Substituting $\nabla_{x_{2 b}}=\partial_{x_{2 b}}$ and $\nabla_{t_{2 b}}=\partial_{t_{2 b}}-\partial_{x_{2 b}} \xi_{0,-1}^{00}$ into the rest of them, we obtain (3.5). q.e.d.

Remark. (3.5) or (3.6) is not stable under the time evolutions.

In fact, we have

Proposition 11. Suppose that $\xi$ satisfies the system of Eqs. (2.1a), (2.1b), (2.1c), and (2.3) and corresponds to a self-dual (anti-self-dual) field on 4 . Then

$$
\begin{gathered}
\text { if } p+q \geqq i-k, k \geqq 0, r+s>j-l, \quad \text { then } \quad \partial_{x_{11}}^{p} \partial_{x_{12}}^{q} \partial_{x_{11}}^{r} \partial_{x_{12}}^{s} \xi_{k l}^{i j}=0, \\
\text { (if } \left.p+q>i-k, r+s \geqq j-l, l \geqq 0, \text { then } \partial_{x_{11}}^{p} \partial_{x_{12}}^{q} \partial_{x_{11}}^{r} \partial_{x_{12}}^{s} \xi_{k l}^{i j}=0\right) .
\end{gathered}
$$


Under the conditions (2.1a), (2.1b), and (2.1c), Eqs. (3.7) are equivalent to the following equations:

$$
\begin{aligned}
& \text { If } p+q \geqq i \text { and } r+s>-l \text {, then } \partial_{x_{11}}^{p} \partial_{x_{12}}^{q} \partial_{x_{21}}^{r} \partial_{x_{22}}^{s} \xi_{0 l}^{i 0}=0 . \\
& \text { (If } p+q>-k \text { and } r+s \geqq j, \text { then } \partial_{x_{11}}^{p} \partial_{x_{12}}^{q} \partial_{x_{21}}^{r} \partial_{x_{22}}^{s} \xi_{k 0}^{0 j}=0 . \text {.) }
\end{aligned}
$$

Proof. By using (2.8), the proposition can be reduced by induction to the case $p=q=i=k=0$. (Note that $\xi_{k l}^{i j}=\xi_{0, l}^{i-k, j}$ if $k \geqq 0$.) Moreover, by using (2.2), it can be reduced to the case $j=0$, i.e. $\partial_{x_{21}}^{r} \partial_{x_{22}}^{s} \xi_{0 l}^{00}=0$ if $r+s>-l$, or $\partial_{x_{21}}^{r} \partial_{x_{22}}^{s} w_{0 j}=0$ if $r+s>j$. By using the second equation of (1.9), it can be reduced to the case $j=1$ which is nothing but (3.6). This completes the proof. (The latter half of the statement can be proved as in Proposition 5.)

Theorem 3. A solution $\xi$ to the system of Eqs. (2.1a), (2.1b), (2.1c), and (2.3) corresponds to a self-dual (anti-self-dual) field on $\Delta$ if and only if its initial datum $\xi^{(0)}$ satisfies (3.7).

Proof. Suppose that $\xi^{(0)}$ satisfies (3.7). Differentiating both sides of (2.11), we can see that $\tilde{\xi}$ also satisfies (3.7):

$$
\text { If } p+q \geqq i-k, k \geqq 0, r+s>j-l, \quad \text { then } \partial_{x_{11}}^{p} \partial_{x_{12}}^{q} \partial_{x_{21}}^{r} \partial_{x_{22}}^{s} \tilde{\zeta}_{k l}^{i j}=0 \text {. }
$$

Set

and

$$
\begin{aligned}
& \mathscr{R}_{3}=\left\{A \in \mathscr{R}_{2} \mid A \text { satisfies }(3.7)\right\}, \\
& \mathscr{F}_{3}=\left\{\xi \in \mathscr{F}_{2} \mid \xi \text { satisfies }(3.7)\right\}
\end{aligned}
$$

$$
\mathscr{\mathscr { F }}_{3}[[t]]=\left\{\sum_{i \geqq 0} \xi_{t} t^{i} \mid \xi_{i} \in \mathscr{F}_{3}\right\} .
$$

Then $\mathscr{R}_{3}$ is a subring of $\mathscr{R}_{2}$ and $\mathscr{F}_{3}$ is an $\mathscr{R}_{3}$-module. It follows from (3.9) that $\tilde{\zeta} \in \mathscr{F}_{3}[[t]], \tilde{\zeta}_{(-)}, \tilde{\zeta}_{(-)}^{-1} \in \mathscr{R}_{3}[[t]]$. Therefore $\xi=\widetilde{\xi}_{\xi} \cdot \widetilde{\xi}_{(-)}^{-1} \in \mathscr{F}_{3}[[t]]$. q.e.d.

\section{Special Solutions}

Proposition 12. Let $w(\lambda) \leftrightarrow \xi$ through the correspondence in Proposition 4, $w^{(0)}(\lambda)=\left.w(\lambda)\right|_{t=0}$ and $\xi^{(0)}=\left.\xi\right|_{t=0}$. Suppose that $w(\lambda)$ satisfies (1.9). Then the following (i), (ii), (iii), and (iv) are equivalent one another for any $p, q \in \mathbb{N}$ :

(i) If $i>p$ or $j>q$, then $w_{i j}^{(0)}=0$.

(ii) If $i>p$ or $j>q$, then $w_{i j}=0$.

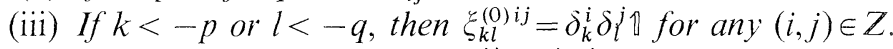

(iv) If $k<-p$ or $l<-q$, then $\xi_{k l}^{i j}=\delta_{k}^{i} \delta_{l}^{j} \mathbb{1}$ for any $(i, j) \in Z$.

Proof.

Proof that (ii) implies (iv). When $(i, j) \in N^{c}$, (iv) is trivially satisfied because of (2.1a). Therefore we assume that $(i, j) \in N$. If $k<-p$, then $\xi_{k l}^{i j}=-\sum_{(g, h) \in N} w_{i-g, j-h}^{*} w_{g-k, h-l}$ $=0$ because $g-k \geqq-k>p$ for $g \geqq 0$. If $l<-q$, then $\xi_{k l}^{i j}=-\sum_{(g, h) \in N} w_{i-g, j-h}^{*} w_{g-k, h-l}$ $=0$ because $h-l \geqq l>q$ for $h \geqq 0$. 
Proof that (iv) implies (ii). If $i>p$ or $j>q$, then $w_{i j}=-\xi_{-i,-j}^{0,0}=0$ because $-i<-p$ or $-j<-q$.

Equivalence of (i) to (iii). This can be proved similarly.

Equivalence of (iii) to (iv). It is obvious that (iv) implies (iii). We shall prove that (iii) implies (iv). Let

and

$$
\begin{gathered}
\mathscr{R}_{4}=\left\{A \in \mathscr{R}_{1} \mid A \text { satisfies }(2.6) \quad\right. \text { and } \\
\left.A_{k l}^{i j}=\delta_{k}^{i} \delta_{l}^{j} \text { if } i \geqq k<-p \quad \text { or } j \geqq l<-q\right\}, \\
\mathscr{F}_{4}=\left\{\xi \in \mathscr{F}_{1} \mid \xi \text { satisfies }(2.6)\right. \text { and } \\
\left.\xi_{k l}^{i j}=\delta_{k}^{i} \delta_{l}^{j} \text { if } i \geqq k<-p \quad \text { or } \quad j \geqq l<-q\right\}
\end{gathered}
$$

$$
\mathscr{F}_{4}[[t]]=\left\{\sum_{l \geqq 0} \xi_{t} t^{i} \mid \xi_{i} \in \mathscr{F}_{4}\right\} .
$$

Then $\mathscr{R}_{4}$ is a subring of $\mathscr{R}_{1}$ and $\mathscr{F}_{4}$ is a right $\mathscr{R}_{4}$-module on which $\Lambda_{a} \partial_{x_{a b}}(a, b=1,2)$ act. If $\xi^{(0)} \in \mathscr{F}_{4}$, then $\tilde{\xi}=\sum_{p \geqq 0} \frac{1}{p !}\left(\sum_{a=1}^{2} \sum_{b=1}^{2} t_{a b} \Lambda_{a} \partial_{x_{a b}}\right)^{p} \xi^{(0)} \in \mathscr{F}_{4}[[t]]$, and hence $\tilde{\xi}_{(-)} \in \mathscr{R}_{4}[[t]]$. Therefore $\xi=\tilde{\xi}\left(\tilde{\xi}_{(-)}\right)^{-1} \in \mathscr{F}_{4}[[t]]$. This completes the proof.

Thus, starting from an initial value $w^{(0)}(\lambda)$ which is a polynomial of $\lambda_{1}^{-1}, \lambda_{2}^{-1}$, we obtain such a solution.

Now we shall illustrate a simplest non-trivial example. Let

$$
w^{(0)}(\lambda)=1+w_{10}^{(0)} \lambda_{1}^{-1}+w_{01}^{(0)} \lambda_{2}^{-1} .
$$

Then $\xi_{0 l}^{(0) i 0}=-\sum_{g=0}^{i} w_{i-g, 0}^{(0) *} w_{g,-l}^{(0)}=-w_{i 0}^{(0)} * w_{01}^{(0)}$ if $i \geqq 0$ and $l=-1$, and $\xi_{0 l}^{(0) i 0}=0$ otherwise. $\xi_{k 0}^{(0) 0 j}=-\sum_{h=0}^{j} w_{0, j-h}^{(0) *} w_{-k, h}^{(0)}=-w_{0 j}^{(0) *} w_{10}^{(0)}$ if $j \geqq 0$ and $k=-1$, and $\xi_{k 0}^{(0) 0 j}=0$ otherwise. We can see that $w_{i 0}^{(0)} *=\left(-w_{10}^{(0)}\right)^{i}$ and that $w_{0 j}^{(0)} *=\left(-w_{01}^{(0)}\right)^{j}$ because $w(\lambda)^{-1}$ $=\sum_{i, j \geqq 0}\left(\begin{array}{c}i+j \\ i\end{array}\right)\left(-w_{10}^{(0)}\right)^{i}\left(-w_{01}^{(0)}\right)^{j} \lambda_{1}^{-i} \lambda_{2}^{-j}$. Therefore (2.7) is written as

$$
\begin{aligned}
& \partial_{x_{11}}^{p} \partial_{x_{12}}^{i+1-p}\left\{\left(w_{10}^{(0)}\right)^{i} w_{01}^{(0)}\right\}=0 \text { for } p=0,1, \ldots, i+1, \\
& \partial_{x_{21}}^{q} \partial_{x_{22}}^{j+1-q}\left\{\left(w_{01}^{(0)}\right)^{j} w_{10}^{(0)}\right\}=0 \text { for } q=0,1, \ldots, j+1 .
\end{aligned}
$$

Since (4.1) reads $\partial_{x_{11}} w_{01}^{(0)}=\partial_{x_{12}} w_{01}^{(0)}=\hat{\partial}_{x_{21}} w_{10}^{(0)}=\partial_{x_{22}} w_{10}^{(0)}=0$ when $i=j=0$, the gauge field corresponds to a self-dual Yang-Mills field on $\Delta$ if and only if

$$
\partial_{x_{21}}^{2} w_{01}^{(0)}=\partial_{x_{21}} \hat{\partial}_{x_{22}} w_{01}^{(0)}=\partial_{x_{22}}^{2} w_{01}^{(0)}=0
$$

and corresponds to an anti-self-dual Yang-Mills field if and only if

$$
\hat{c}_{x_{11}}^{2} w_{10}^{(0)}=\partial_{x_{11}} \partial_{x_{12}} w_{10}^{(0)}=\partial_{x_{12}}^{2} w_{10}^{(0)}=0 \text {. }
$$

Now set $w_{10}^{(0)}=c_{11} x_{11}^{2}+c_{12} x_{12}^{2}, w_{01}^{(0)}=c_{21} x_{21}^{2}+c_{22} x_{22}^{2}, c_{a b} \in M_{n}(\mathbb{C})$ for $a, b=1,2$. Then (4.1) is satisfied for $i=j=0$. Equation (4.2) is equivalent to $c_{11}=c_{12}=0$ and (4.3) is equivalent to $c_{21}=c_{22}=0$. On the other hand, noting that $A_{t_{a b}}^{(0)}=2 c_{a b} x_{a b}$ for 
$a, b=1,2$, we see that if the gauge field $\nabla$ is abelian, then $\left[c_{a b}, c_{d e}\right]=0$ for $a, b, d, e$ $=1,2$. Note that the gauge field $\nabla$ corresponds to an abelian Yang-Mills field on $\Delta$ if and only if $\nabla$ is abelian itself. Now set, for example,

$$
\begin{aligned}
& c_{11}=E_{21}=\left(\delta_{i 2} \delta_{j 1}\right)_{i=1, \ldots, n, j=1, \ldots, n,} \\
& c_{12}=E_{32}=\left(\delta_{i 3} \delta_{j 2}\right)_{i=1, \ldots, n, j=1, \ldots, n,}, \\
& c_{22}=E_{54}=\left(\delta_{i 5} \delta_{j 4}\right)_{i=1, \ldots, n, j=1, \ldots, n,} \\
& c_{22}=E_{65}=\left(\delta_{i 6} \delta_{j 5}\right)_{i=1, \ldots, n, j=1, \ldots, n} .
\end{aligned}
$$

Then $w_{01}^{(0)} w_{10}^{(0)}=w_{10}^{(0)} w_{01}^{(0)}=0$ and especially (4.1) is satisfied for $i \geqq 0$ and $j \geqq 0$. Neither (4.2) nor (4.3) holds because $c_{a b} \neq 0$ for $a, b=1,2$. The gauge field is not abelian because $\left[c_{11}, c_{12}\right],\left[c_{21}, c_{22}\right] \neq 0$. Thus we obtain a gauge field corresponding to a Yang-Mills field on $\Delta$ which is not abelian, self-dual nor anti-self-dual.

\section{References}

1. Chau, L.L., Prasad, M.K., Sinha, A.: Some aspects of the linear systems for self-dual YangMills fields. Phys. Rev. D24, 1574-1580 (1981)

2. Isenberg, J., Yasskin, P. B., Green, P.S. : Non-self-dual gauge fields. Phys. Lett. B78, 462-464 (1978)

3. Pohlmeyer, K.: On the Lagrangian theory of anti-self-dual fields in four dimensional Euclidean space. Commun. Math. Phys. 72, 37-47 (1980)

4. Sato, M.: Soliton equations as dynamical systems on an infinite dimensional Grassmann manifold. RIMS Kokyuroku 439, 30-46, RIMS, Kyoto University (1981)

5. Sato, M., Sato, Y.: Soliton equations as dynamical systems on infinite dimensional Grassmann manifold. Lecture Notes in Num. Appl. Anal. 5, 259-271 (1982)

6. Suzuki, N.: Structure of the solution space of Witten's gauge-field equations. Proc. Jpn. Acad. 60 A, 141-144 (1984)

7. Takasaki, K.: On the structure of solutions to the self-dual Yang-Mills equations. Proc. Jpn. Acad. 59 A, 418-421 (1983)

8. Takasaki, K. : A new approach to the self-dual Yang-Mills equations. Commun. Math. Phys. 94, 34-59 (1984)

9. Witten, E.: An interpretation of classical Yang-Mills theory. Phys. Lett. B77, 394-398 (1978)

Communicated by H. Araki

Received April 28, 1987 